\title{
Spontaneous rupture of a degenerated leiomyoma causing panperitonitis and ileus during pregnancy: A case report
}

\author{
Mizuha Odagami ${ }^{1}$, Mutsuko Makino $^{1}$, Etsuko Miyagi $^{2}$, and Shigeru Aoki ${ }^{1}$ \\ ${ }^{1}$ Yokohama City University Medical Center \\ ${ }^{2}$ Yokohama City University Hospital
}

July 31,2020

\begin{abstract}
A degenerated leiomyoma rarely ruptures during pregnancy. As the rupture of a degenerated leiomyoma can cause panperitonitis due to the leakage of fluid content into the peritoneal cavity, prompt diagnosis and surgical intervention are required.

Title: Spontaneous rupture of a degenerated leiomyoma causing panperitonitis and ileus during pregnancy: A case report

Authors: Mizuha Odagami ${ }^{1 *}$, Mutsuko Makino $^{1}$, Etsuko Miyagi $^{2}$, Shigeru Aoki $^{1}$

${ }^{1}$ Perinatal Center for Maternity and Neonate, Yokohama City University Medical Center, Yokohama, Japan

2 Department of Obstetrics and Gynecology, Yokohama City University Hospital, Yokohama,

Japan

*Corresponding Author: Mizuha Odagami, M. D.

Perinatal Center for Maternity and Neonate

Yokohama City University Medical Center

4-57 Urafune-cho, Minami-ku, Yokohama City

Kanagawa 232-0024, Japan

Tel: +81-45-261-5656

Fax: +81-45-253-5784

E-mail: mmmizuha@yahoo.co.jp
\end{abstract}

[Key Clinical Message]

A degenerated leiomyoma rarely ruptures during pregnancy. As the rupture of a degenerated leiomyoma can cause panperitonitis due to the leakage of fluid content into the peritoneal cavity, prompt diagnosis and surgical intervention are required.

[Key Words]

Ruptured leiomyoma, degenerated leiomyoma, panperitonitis, myomectomy, pregnancy.

[Introduction] 
Leiomyomas are found in approximately $10 \%$ of pregnant women (1). Pain caused by a degenerated leiomyoma is considered to be the most common complication of pregnancy in women with leiomyomas (2). In many cases, the symptoms of a degenerated leiomyoma improve with conservative therapy; thus, surgical treatment is rarely required $(3,4)$. We report the case of a 38-year-old primiparous woman who underwent a myomectomy during pregnancy as a result of panperitonitis and ileus secondary to the rupture of a degenerated leiomyoma.

[Case]

A 38-year-old primiparous woman was diagnosed with a leiomyoma prior to her pregnancy. At a gestational age of $14+3$ weeks, she had lower abdominal pain and was admitted to a primary health facility with a diagnosis of pain due to a degenerated leiomyoma, for which conservative therapy was started. Because of increased abdominal pain, she was transported to our hospital at a gestational age of $15+0$ weeks. On admission, she complained of severe abdominal pain and vomited several times. In addition to the peritoneal irritation sign, a goose egg-sized mass was palpated in the right upper abdomen, and severe tenderness was observed at the same site. Abdominal ultrasonography revealed an intramural leiomyoma measuring $7 \mathrm{~cm}$ in diameter at the uterine fundus. Blood tests showed the following results: white blood cells (WBC), $12300 / \mathrm{mm}^{3}$; red blood cells, $411 \times 104 / \mathrm{mm}^{3}$; hemoglobin, $13.1 \mathrm{~g} / \mathrm{dL}$; and c-reactive protein $(\mathrm{CRP}), 8.52$ $\mathrm{mg} / \mathrm{dL}$.

Plain abdominal radiography revealed marked dilatation of the small intestine and ileus was diagnosed. Pelvic magnetic resonance imaging (MRI) revealed a leiomyoma with partial high-signal intensity at the uterine fundus; it was considered a degenerated leiomyoma (Fig 1). Based on the above findings, the patient was diagnosed with pain from a degenerated leiomyoma, peritonitis associated with infection of the degenerated leiomyoma, and ileus; hence, the conservative therapy was continued. The patient was afebrile after admission and various culture tests, including blood and urine cultures, were negative, indicating no signs of infection. The levels of inflammatory markers decreased after 1 week (WBC: 9600/ $\mathrm{mm}^{3}$; CRP: $2.3 \mathrm{mg} / \mathrm{dL}$ ). However, because of the poor improvement in abdominal pain and prolonged ileus, it was suspected that the patient had panperitonitis and ileus caused by the rupture of the degenerated leiomyoma and emergency surgery was decided.

The intraoperative examination revealed an intramural leiomyoma measuring $7 \mathrm{~cm}$ in diameter at the uterine fundus. When the omentum and small intestine adhering to the surface of the leiomyoma were dissected, the leiomyoma, with its capsule ruptured, was exposed (Fig 2). Accordingly, the patient was diagnosed with a ruptured, degenerated leiomyoma and a myomectomy was performed. The pathological findings of the resected specimen indicated a leiomyoma with degeneration. After the surgery, the symptoms rapidly improved and the pregnancy course was uneventful. At a gestational age of $37+4$ weeks, elective cesarean delivery was performed because of the myomectomy during pregnancy. The patient delivered a female infant weighing $2870 \mathrm{~g}$, which was appropriate for the gestational age. The Apgar score was 9 at both 1 and 5 minutes. The postoperative course was uneventful.

[Discussion]

This case highlighted the following two findings: first, leiomyomas can rupture during pregnancy. There have been cases reported of the rupture of a blood vessel overlying the leiomyoma during pregnancy $(5,6)$ and after labor (7), resulting in intra-abdominal bleeding; a case of non-reassuring fetal status and intraabdominal bleeding due to the rupture of a leiomyoma during labor has also been reported (4). Thus, the rupture of a leiomyoma can occur at any time. The mechanism of leiomyoma rupture has not been clarified. One of the possible mechanisms is that necrosis associated with the degenerated leiomyoma occurs in the uterine serosa and causes a rupture in the serosa. It is also considered that pregnancy, vigorous exercise, and defecation, which increases abdominal pressure, may cause the congestion of a blood vessel overlying the leiomyoma, resulting in its rupture $(8,9)$. Therefore, the rupture of a leiomyoma should always be kept in mind when treating a pregnancy complicated by a leiomyoma. Second, as the rupture of a leiomyoma causes panperitonitis, it is an indication for prompt surgical treatment. Leiomyoma rupture usually results 
in intra-abdominal bleeding due to the rupture of a blood vessel overlying the leiomyoma, often leading to an acute abdomen $(5,6,9,10)$. However, according to some reports, the rupture of a degenerated leiomyoma caused no intra-abdominal bleeding but caused panperitonitis due to leakage of the fluid content of the degenerated leiomyoma into the peritoneal cavity $(11,12)$. Our case is considered similar to the latter case. Conservative therapy can improve the pain caused by a degenerated leiomyoma but not the symptoms of a ruptured leiomyoma. Therefore, in addition to the pain caused by leiomyoma degeneration, if intraperitoneal bleeding, peritonitis, or ileus is present, leiomyoma rupture should be considered. In such cases, prompt diagnosis and surgical treatment are required to avoid an adverse outcome.

[Conclusion]

A degenerated leiomyoma rarely ruptures during pregnancy. As the rupture of a degenerated leiomyoma can cause panperitonitis, prompt diagnosis and surgical intervention are required.

[Acknowledgment]

We would like to thank HONYAKU CENTER (www.honyakuctr.com) for English language editing.

[Conflict of interest]

None declared.

[Author Contributors]

MO drafted the manuscript contributed and performed the surgery.

MM performed the surgery.

SA contributed to the first draft and finalization of the manuscript.

EM supervised the case report.

[Patient consent]

Obtained

[References]

1. Lee HJ, Norwitz ER, Shaw J. Contemporary management of fibroids in pregnancy. Rev Obstet Gynecol 2010; 3(1):20-27.

2. Katz VL, Dotters DJ, Drogenmuller W. Complications of uterine leiomyomas in pregnancy. Obstet Gynecol 1989; 73(4):593-596.

3. Kobayashi F, Kondoh E, Hamanishi J, Kawamura Y, Tatsumi K, Konishi I. Pyomayoma during pregnancy: A case report and review of the literature. J Obstet Gynaecol Res 2012; 39(1):383-389.

4. Swarray-Deen A, Mensah-Brown SA, Coleman J. Rare complication of fibroids in pregnancy: Spontaneous fibroid rupture. J Obstet Gynaecol Res 2017; 43(9):1485-1488.

5. Kasum M. Hemoperitoneum caused by a bleeding myoma in pregnancy. Acta Clin Croat 2010; 49(2):197-200.

6. Wong L, Ching TW, Kok TL, Koon TH. Spontaneous hemoperitoneum from a uterine leiomyoma in pregnancy. Acta Obstet Gynecol Scand 2005; 84(12):1208-1209.

7. Ramskill N, Hameed A, Beebeejaun Y: Spontaneous rupture of uterine leiomyoma during labor. BMJ Case Rep 2014; 2014:bcr2014204364.

8. Schwartz M, Powell K. Spontaneous rupture of a leiomyoma causing life-threatening intra-abdominal hemorrhage. Case Rep Obstet Gynecol 2017; 2017:1-3.

9. Chen CH, Lin JY, Tzeng CR, Chiu LH, Liu WM. Hemoperitoneum secondary to rupture of a superficial uterine artery overlying a subserous myoma with no predisposing factors in a young woman. Taiwan J Obstet Gynecol 2013; 52(3):133-134. 
10. Fontarensky M, Cassagnes L, Bouchet P, Azuar AS, Boyer L, Chabrot P. Acute complications of benign uterine leiomyomas: Treatment of intraperitoneal haemorrhage by embolisation of the uterine arteries. Diagn Interv Imaging 2013; 94(9):885-890.

11. Makar AP, Meulyzer PR, Vergote IB, Schatteman EA, Huyghe ML, Meeuwis LA, et al. A case report of unusual complication of myomatous uterus in pregnancy: spontaneous perforation of myoma after red degeneration. Eur J Obstet Gynecol Reprod Biol 1989; 31(3):289-293.

12. Kamat NV, Telkar HB, Ramani SK, Thakker AP. Ruptured degenerated uterine fibroid diagnosed by imaging. Obstet Gynecol 2001; 98(5):961-963.

[Figures]

Figure 1: Coronal T2-weighted magnetic resonance imaging showing a leiomyoma with partial high-signal intensity at the uterine fundus, which was considered to be a degenerated leiomyoma.

Figure 2: Intraoperative examination revealed an intramural leiomyoma at the uterine fundus, with the upper half of its capsule ruptured.

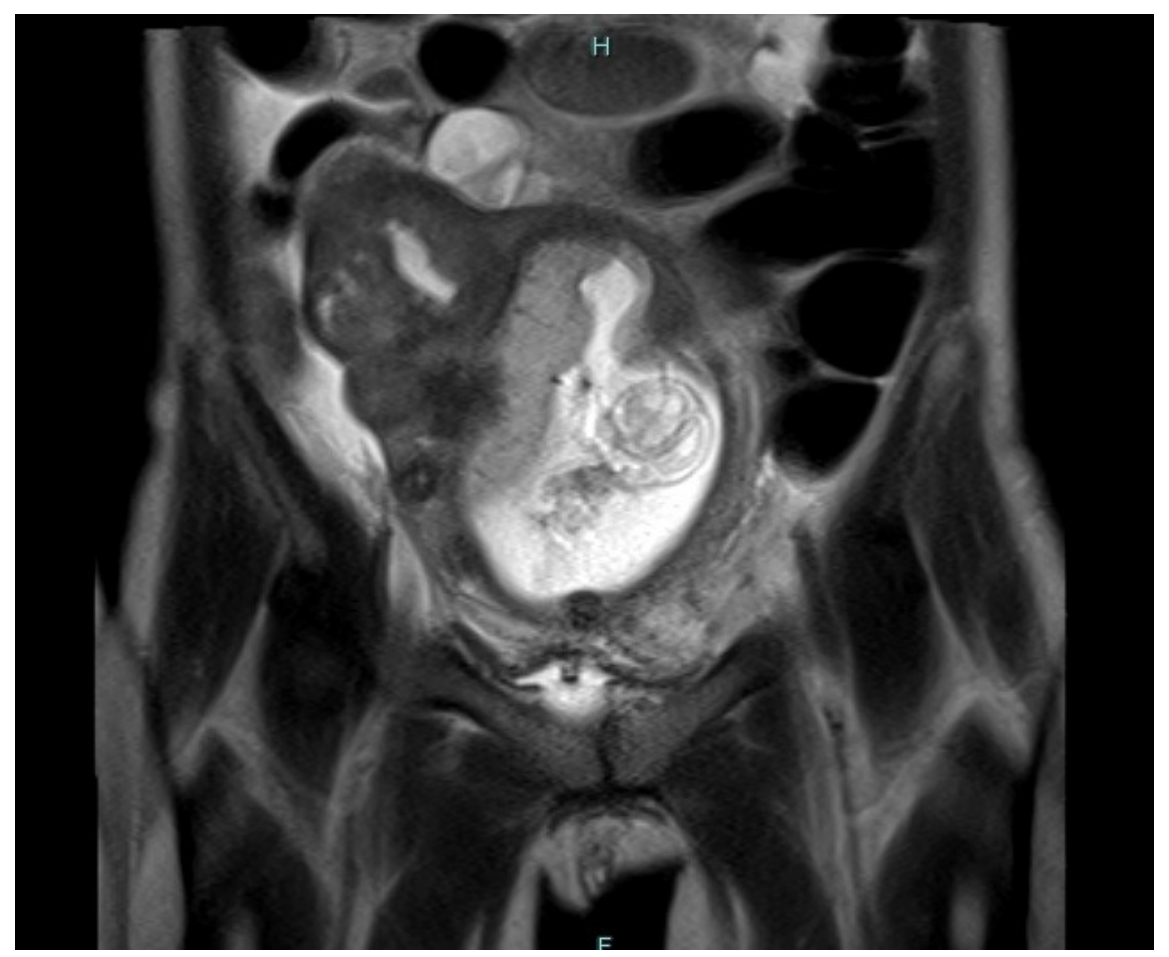




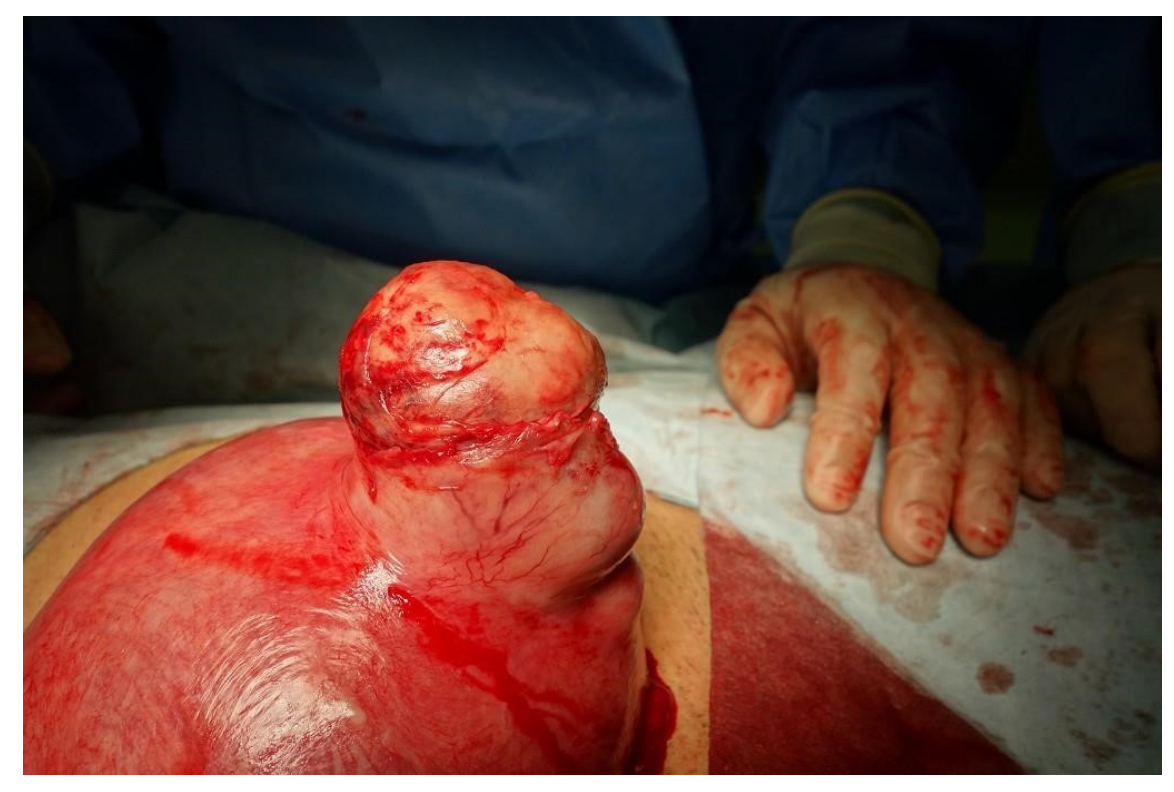

